\title{
The Potential of the Timing Method to Detect Evolved Planetary Systems
}

\author{
Roberto Silvotti*, Róbert Szabó ${ }^{\dagger}$, Pieter Degroote**, Roy H. Østensen** \\ and Sonja Schuh ${ }^{\ddagger}$ \\ *INAF-Osservatorio Astronomico di Torino, strada dell'Osservatorio 20, \\ 10025 Pino Torinese, Italy \\ ${ }^{\dagger}$ Konkoly Observatory of the Hungarian Academy of Sciences, Konkoly Thege Miklós út 15-17, \\ 1121 Budapest, Hungary \\ ${ }^{* *}$ Instituut voor Sterrenkunde, K.U. Leuven, Celestijnenlaan 200D, 3001 Leuven, Belgium \\ ${ }^{\ddagger}$ Georg-August-Universität Göttingen, Institut für Astrophysik, Friedrich-Hund-Platz 1, \\ 37077 Göttingen, Germany
}

\begin{abstract}
The timing method, using either stellar pulsations or eclipse timing of close binaries as a clock, is proving to be an efficient way to detect planets around stars that have evolved beyond the red giant branch. In this article we present a short review of the recent discoveries and we investigate the potential of the timing method using data both from ground-based facilities as well as from the Kepler and CoRoT space missions.
\end{abstract}

Keywords: exoplanets, post-MS stars, stellar pulsation

PACS: 97 (97.10.Sj, 97.20.Rp, 97.20.Tr, 97.30.Dg, 97.30.Kn, 97.80.Hn, 97.82.Fs)

\section{INTRODUCTION}

The large majority of the almost 500 extrasolar planets detected so far ${ }^{1}$ orbit main sequence (MS) stars, and these planets were detected mostly using radial velocities (RVs) or transits. For various reasons, not only technical, current exoplanet research is strongly oriented towards MS stars, with the main goal of finding rocky analogs of our Earth, and towards understanding planetary system formation, while the latestage evolution after the MS remains poorly understood. The first attempts to model the evolution of the planetary systems after the red giant phase are very recent (Villaver \& Livio 2009 and ref. therein), and in particular lack observational constraints. The two main methods to detect exoplanets, RVs and transits, are not efficient for compact stars with high gravities and small radii, such as the extreme horizontal branch stars and the white dwarfs. An alternative method for these stars is given by the timing method, in analogy with the first pulsars' planet discoveries (Wolszczan \& Frail 1992). This method is based on the reflex motion of the parent star due to the companion, which changes periodically the star-observer distance, causing a delay (or advance) on the arrival time of the photons. In order to use the timing method, what we need is a precise astrophysical clock. For the pulsars' planets, this is given by the pulsar's radio signal modulated by the fast and stable rotation of the neutron star. But other potentially precise clocks exist

1494 from the Extrasolar Planets Encyclopaedia (http: //exoplanet.eu/) on October 12th, 2010. 
in astrophysics and are given, for example, by stellar pulsation timing or the eclipse timing in close binary systems. An early example of application of the timing method is given by Paparó et al. (1988), who calculated the orbital elements of an unseen stellar companion to the Delta Scuti star SZ Lyncis using the O-C technique (Sterken 2005) on the pulsation timing. The method has also been discussed for pulsating white dwarfs by Kepler et al. (1991) and Provencal (1997).

The next sections contain an introduction to the field of post-RGB planetary systems and a short review of the recent discoveries, respectively. Then we investigate the potential of the timing method to find new planetary systems around evolved stars using pulsation or eclipse timing, with particular attention to the ongoing space missions CoRoT and Kepler. Finally, in the last section, a few conclusions are given and future perspectives are discussed.

\section{POST-RGB EVOLUTION OF PLANETARY SYSTEMS}

During the red giant (RG) expansion of a star, two main opposite phenomena influence the orbits of potential planetary companions: mass loss from the star, which lowers the binding energy and hence tends to cause an outward drift of the planet, and tidal effects, which tend to attract the planet close to the star and eventually inside the envelope. If the planet enters the stellar envelope, it is not clear in which conditions it can survive and what can be the influence on the subsequent evolution of the star. On the other hand, we know that during the RG phase a small fraction of stars, of the order of $1 \%$, loose almost all their envelope and are left with a very thin $\mathrm{H}$ envelope during their core $\mathrm{He}$ burning phase. These are the so-called subdwarf $\mathrm{B}$ (or sdB) stars, that are located on the extreme horizontal branch (EHB), at high effective temperatures ( $\gtrsim 20,000 \mathrm{~K})$. At least $50 \%$ of them are in binary systems and in this case the formation of an sdB star can be explained in terms of binary evolution (Han et al. 2002, 2003). However, for the remaining fraction of apparently single sdB stars, it is more difficult to explain why the huge mass loss during the RGB occurred (see review by Heber 2009). In this context planets might play a role (Soker 1998).

Similar phenomena of planetary migration, planet engulfment and planet accretion from stellar ejected material can happen also during the second large expansion of a star, when it enters the asymptotic giant branch and the thermal pulses phase, and during the planetary nebula (PN) ejection.

In order to achieve consistent modelling of all these complex phenomena, tests against observational constraints are essential. To detect planets around white dwarfs is (or more exactly will be) a key step to study the late-stage evolution of planetary systems. Equally important is to identify planetary systems in previous shorter and more rare evolutionary phases like the RGB or the horizontal branch. Only in this way can we try to disentangle the various phenomena that changed the configuration of the planets and their orbits, with the final goal of being able to trace back the entire history of a planetary system from the final white dwarf configuration to the original protoplanetary disk. What we need first is to find a sufficient number of planetary systems orbiting post-RGB stars and white dwarfs, covering a wide range of orbital parameters and planetary masses.

Concerning white dwarfs, what we know already is that some of these stars are 
surrounded by metal-rich debris disks: at least 17 white dwarfs show circumstellar dust at $\sim 1,000 \mathrm{~K}$ (Reach et al. 2005 and ref. therein, Farihi et al. 2010) and about 20\% of them show also Ca II emission from a hotter gaseous disk (Gänsicke et al. 2006, 2010). These disks are generally very close to the white dwarf, within about $1 \mathrm{R}_{\odot}$. Recently, also much more distant and cooler debris disks have been detected ( $\mathrm{Su}$ et al. 2007, Chu et al. 2010 and Bilíková et al. 2010), which are probably the final evolution of the solar system's Kuiper belt analogues. In other words, we don't know yet how many white dwarfs host planets and at which distances from the star, but we know that at least the material to build planets is there.

Another interesting possibility is that second (or even third) generation planets can form from the stellar material ejected during stellar evolution (Perets et al. 2010). But again, to confirm these theoretical scenarios, we need observations and statistics.

\section{RECENT DETECTIONS OF POST-RGB PLANETARY SYSTEMS}

From the observational side, the late-stage evolution of the planetary systems is a very recent field and only in the last three years a few first post-RGB planetary systems were detected. They belong mainly to two classes:

1. Extreme horizontal branch hot subdwarfs B:

V391 Peg b (Silvotti et al. 2007),

HW Vir b and c (Lee et al. 2009),

HS 0705+67003 b (Qian et al. 2009a),

HD 149382 b (Geier et al. 2009, but see also Jacobs et al. 2010).

2. Cataclysmic variables:

NN Ser b and c (Qian et al. 2009b, Beuermann et al. 2010a, Hessman et al. 2010), DP Leo b (Qian et al. 2010a, Beuermann et al. 2010b) and

QS Vir b (Qian et al. 2010b).

We note that the issue of nomenclature for circumbinary planets, in contrast to the nomenclature for planets around components of visually resolved binaries, is unsolved. On recommendation by the $A \& A$ editor, Beuermann et al. (2010a, 2010b) follow the rule by which any unseen companions, whether stellar or sub-stellar, are designated by lowercase letters. They hence have introduced $(\mathrm{ab})$ for the two components of the central binary and start numbering the third bodies with the letter c, resulting in the planet designations NN Ser (ab) c, NN Ser (ab) d, and DP Leo (ab) c.

Another interesting new planet candidate was presented during this meeting by Setiawan et al. 2010a (a 1.3 $\mathrm{M}_{\text {Jup }}$ planet at $0.116 \mathrm{AU}$ from a red HB star of likely extragalactic origin).

In Table 1 we summarize the list of known post-RGB planets, including the pulsars' planets and a few brown dwarfs (BDs) near the planet mass limit. We include also the brown dwarf WD $0137-349$ b because its very small orbital distance, only $0.375 \mathrm{R}_{\odot}$, implies that the BD was engulfed by the red giant progenitor of the white dwarf (Maxted et al. 2006). As one can see, most of the detections reported in Table 1 were obtained through timing measurements. 
TABLE 1. Post-RGB planets

\begin{tabular}{|c|c|c|c|c|c|c|c|c|}
\hline \multirow{2}{*}{$\frac{\text { Planet }}{\text { PSR 1257+12b }}$} & \multicolumn{2}{|c|}{$\mathbf{M} \sin \mathbf{i}\left[\mathbf{M}_{\mathrm{Jup}}\right]$} & \multirow{2}{*}{$\frac{\mathbf{a}[\mathbf{A U}]}{0.19}$} & $\mathbf{P}[\mathbf{y r}]$ & \multirow{2}{*}{$\frac{\mathbf{e}}{0}$} & \multirow{4}{*}{$\begin{array}{l}\text { Evolut. phase } \\
\text { pulsar }\end{array}$} & \multirow{4}{*}{$\begin{array}{l}\text { Detection method } \\
\text { timing (radio signal) } \\
\text { timing (radio signal) } \\
\text { timing (radio signal) }\end{array}$} & \multirow{4}{*}{$\begin{array}{l}\text { References } \\
\text { Wolszczan \& Frail } 1992 \\
\text { Konacki \& Wolszczan } 2003\end{array}$} \\
\hline & $M \simeq$ & $6 e-5$ & & $25.262 \mathrm{~d}$ & & & & \\
\hline PSR $1257+12 c$ & $M \simeq$ & 0.014 & 0.36 & $66.5419 \mathrm{~d}$ & 0.0186 & & & \\
\hline PSR $1257+12 \mathrm{~d}$ & $M \simeq$ & 0.012 & 0.46 & $98.2114 \mathrm{~d}$ & 0.0252 & & & \\
\hline PSR B1620-26b & & 2.5 & 23 & 100 & & pulsar+WD & timing (radio signal) & Thorsett et al. 1993 \\
\hline & & & & & & in globular cluster & & Sigurdsson et al. 2003 \\
\hline WD 0137-349b & $M \simeq$ & 55 & $0.375 \mathrm{R}_{\odot}$ & $1.927 \mathrm{~h}$ & & WD & $\mathrm{RVs}$ & Maxted et al. 2006 \\
\hline GD 66 b (?) & $M<7:$ & 2.4 & 2.75 & 5.7 & 0 & WD (DAV) & timing (pulsations) & Mullally et al. 2008, 2009 \\
\hline GD 356 b (?) & $M<12$ & & & $>2.7$ & & WD, magnetic & $\begin{array}{l}\text { inferred from } \\
\text { Zeeman splitting }\end{array}$ & Wickramasinghe et al. 2010 \\
\hline V391 Peg b & & 3.2 & 1.7 & 3.2 & 0 & $\mathrm{EHB}\left(\mathrm{sdBV}_{\mathrm{rs}}\right)$ & timing (pulsations) & Silvotti et al. 2007 \\
\hline HW Vir b & & 19.2 & 5.3 & 15.8 & 0.46 & EHB (ecl. sdB+M) & timing (eclipse) & Lee et al. 2009 \\
\hline HW Vir c & & 8.5 & 3.6 & 9.1 & 0.31 & & timing (eclipse) & \\
\hline HS 0705+67003b & & 39.5 & $<3.6$ & 7.15 & & EHB (ecl. sdB+M) & timing (eclipse) & Qian et al. 2009a \\
\hline HD 149382 b (?) & $M=8$ & -23 & $5-6.1 \quad \mathrm{R}_{\odot}$ & $2.391 \mathrm{~d}$ & & EHB (sdB) & RVs & $\begin{array}{l}\text { Geier et al. } 2009 \\
\text { Jacobs et al. } 2010\end{array}$ \\
\hline NN Ser b* & & 6.9 & 5.4 & 15.5 & 0 & pre-CV & timing (eclipse) & Qian et al. 2009b \\
\hline NN Ser c* & & 2.3 & 3.4 & 7.7 & 0.2 & & timing (eclipse) & $\begin{array}{l}\text { Beuermann et al. } 2010 \text { a } \\
\text { Hessman et al. } 2010\end{array}$ \\
\hline DPLeo $^{\dagger}$ & & 6.05 & 8.2 & 28.0 & 0.39 & $\mathrm{CV}$ (polar) & timing (eclipse) & $\begin{array}{l}\text { Qian et al. 2010a } \\
\text { Beuermann et al. 2010b }\end{array}$ \\
\hline QS Vir b & $M \simeq$ & $6.65^{* *}$ & 4.2 & 7.86 & 0.37 & $\mathrm{CV}$, hibernating & timing (eclipse) & Qian et al. 2010b \\
\hline HIP 13044 b & & 1.25 & 0.116 & 16.2 & 0.25 & $\begin{array}{l}\text { RHB } \\
\text { (extragal. orig.?) }\end{array}$ & RVs & $\begin{array}{l}\text { Setiawan et al. 2010a } \\
\text { Setiawan et al. } 2010 \mathrm{~b}\end{array}$ \\
\hline
\end{tabular}

* All numbers given are for the model 2a solution by Beuermann et al. 2010a

$\dagger$ All numbers given are from Beuermann et al. 2010b

** If coplanar to the eclipsing binary 
A circumbinary planet with a minimum mass of about $5 \mathrm{M}_{\text {Jup }}$ could be the reason of the periodic component in the $\mathrm{O}-\mathrm{C}$ plot of the eclipsing polar HU Aquarii (Schwarz et al. 2009, not included in Table 1). O'Toole et al. (2010) also report early results from their on-going RV survey. We refer to their proceeding papers for more details.

For what concerns the white dwarfs, until now there are no clear detections of planets around single white dwarfs, with only two candidates: GD 66 (Mullally et al. 2008, 2009) and GD 356 (Wickramasinghe et al. 2010). We know at least three planet hosting MS stars having a wide binary WD companion: HD 13445=Gl 86, HD 147513 and HD 27442 (Desidera \& Barbieri 2007 and ref. therein). In all these three systems the planet orbit the MS star at an orbital distance much smaller than the WD distance: 0.11 versus $19 \mathrm{AU}$ for HD 13445, 1.26 versus at least $100 \mathrm{AU}$ for HD 147513, and 1.18 versus $240 \mathrm{AU}$ for HD 27442. Therefore it is unlikely that the WD had an influence on the evolution of the planetary system and this is why these systems are not included in Table 1. More interesting (although quite exotic) is the case of the circumbinary planet orbiting the pulsar+WD system PSR 1620-26 (Thorsett et al. 1999, Sigurdsson et al. 2003) in the globular cluster M4. Following Sigurdsson et al. (2003), the most likely scenario is that the planet was initially in orbit around the MS star progenitor of what is now the WD companion. The system underwent an exchange interaction with a neutron star binary, with the MS star replacing the original high-mass WD companion to the neutron star. The end results were a new eccentric orbit for the MS star, and the displacement of the planet onto a wide circumbinary orbit.

\section{ONGOING PROJECTS AND THE POTENTIAL OF COROT AND KEPLER DATA}

Similarly to the astrometric method (and contrary to the RV and transit methods), the timing method is sensitive to large orbital separations. Equation (1) shows that the (maximum) change in the arrival time of the photons (or time shift) $\tau$ is indeed proportional to the orbital distance $a\left(m_{p}, M_{\star}, c\right.$ and $i$ are, respectively, planet mass, stellar mass, light speed and inclination of the orbit to the line of sight):

$$
\tau=\frac{a}{c} \frac{m_{p} \sin i}{M_{\star}}
$$

For $a=1 \mathrm{AU}, m_{p}=1 \mathrm{M}_{\mathrm{Jup}}, M_{\star}=1 \mathrm{M}_{\odot}\left(\right.$ and $\left.i=90^{\circ}\right)$, the time shift is about 0.5 seconds; while it is $1 \mathrm{~s}$ for a stellar mass of $0.5 \mathrm{M}_{\odot}$, as in the case of a typical sdB star or a slightly lower-than-average-mass white dwarf.

As shown by the recent discoveries in the previous section, it is possible to detect planets with the timing method using ground-based data. What is needed is a longterm photometric monitoring of the targets for many years with frequent runs, ideally each month or two. A few ground-based programs to search for post-RGB planets exist, e.g. the EXOTIME program (Schuh et al. 2009, Schuh 2010, Lutz et al. 2010²) which

2 see also http://www.na.astro.it/ silvotti/exotime/ 
is monitoring five sdB pulsators, a similar program at the McDonald Observatory on $15 \mathrm{ZZ}$ Ceti pulsators (Mullally et al. 2008), and the long-term monitoring of one DBV pulsator by Dalessio et al. (2010). A secondary goal of these programs is the measurement of the secular variation of the pulsation periods, which is related to the change of the stellar structure because of its evolution (e.g. Kepler et al. 2005, Costa $\&$ Kepler 2008, Silvotti et al. 2007). Similar ground-based programs exist also for the eclipsing binaries.

However it is clear that, potentially, the maximum results can be achieved using continuous monitoring from space craft. The exceptional photometric precision and duty cycle of the CoRoT and Kepler satellite data represent a unique opportunity to use the timing method to detect low mass substellar companions to pulsating stars and eclipsing binaries. In particular, Kepler has a longer coverage, up to 3.5 years, as the satellite will observe the same $\sim 105$ square degree field for the whole mission duration; while the CoRoT "long runs" have a maximum duration of about 6 months. For more details concerning the Kepler and CoRoT missions we refer the reader to Borucki et al. (2010), Gilliland et al. (2010) and Auvergne et al. (2009). The main limitation of Kepler is the relatively long sampling time of $58 \mathrm{~s}$ (short-cadence data). Although the photometer samples the field every $6.54 \mathrm{~s}$, telemetry restrictions do not permit to save and transmit to the ground the original data. This is a severe limitation only for the very rapid pulsators like the short-period $\mathrm{sdB}$ stars of the $\mathrm{sdBV}_{\mathrm{r}}$ class and the white dwarfs, which can have pulsation periods as short as $\approx 1-2$ minutes. However, in the survey phase (1st year of observation) that has just ended, no pulsating white dwarfs were found and only one short-period sdB pulsator was observed (Kawaler et al. 2010) plus an hybrid pulsator with a very rich amplitude spectrum in a eclipsing binary system with a M dwarf (Østensen et al. 2010a, see also end of this section on 5. Eclipse timing).

For all the other 13 long-period $\mathrm{sdB}$ pulsators $\left(\mathrm{sdBV}_{\mathrm{s}}\right)$ discovered by Kepler (Østensen et al. 2010b, 2010c), with typical periods between 30 min to few hours, the $58 \mathrm{~s} \mathrm{sam-}$ pling is largely sufficient, even though longer pulsation periods mean higher minimum detectable mass of a substellar companion. This is shown by the following equation (2), in which $\sigma_{\tau}$ is the timing error, $N$ is the number of data points, $P$ the pulsation period, $\sigma_{I}$ the photometric (relative intensity) error, $A$ the pulsation amplitude and $\sigma_{A}$ the amplitude error, which decreases with the square root of the length of the run and can be estimated directly by measuring the mean noise of the Fourier amplitude spectrum. Equation (2) is valid under the assumption of uncorrelated errors and no cross terms between the different pulsation frequencies, and is obtained multiplying by $P$ equation (A15) from Breger et al. 1999 (see also Montgomery \& O’Donoghue 1999 and Silvotti et al. 2006 for more details).

$$
\sigma_{\tau}=\frac{P}{2 \pi} \sqrt{\frac{2}{N}} \frac{\sigma_{I}}{A}=\frac{P}{2 \pi} \frac{\sigma_{A}}{A}
$$

A similar equation can be obtained also for the eclipse timing (Doyle \& Deeg 2002), assuming a simple model of triangular eclipse (which implies that the error in eclipse minimum timing $\sigma_{\tau}$ is a lower limit to the real error). In the following equation (3), $T_{\mathrm{ecl}}$ is the duration of the eclipse from first to last contact, $\Delta I$ is the relative intensity of the 
eclipse (or $(1-\Delta I)$ is the eclipse's relative depth, with $I=1$ out of eclipse) and $N_{\text {ecl }}$ is the number of data points taken during $T_{\mathrm{ecl}} ; \sigma_{I}$ was defined above.

$$
\sigma_{\tau}=\frac{T_{\mathrm{ecl}}}{2 \sqrt{N_{\mathrm{ecl}}}} \frac{\sigma_{I}}{(1-\Delta I)}
$$

As we do not expect to find close-in planets with short orbital periods around postRGB stars $^{3}$, we will need many months/years of Kepler data to be able to set limits on the presence of planets around these stars. At the moment, with the available Kepler data, we can only start making some tests to see what the typical phase errors are that we can obtain and therefore what we can expect in terms of minimum detectable masses and orbital periods. The first goal of these preliminary tests is to verify the phase stability (or "phase coherence") of different classes of pulsating stars and eclipsing binaries.

Regarding the pulsators, the most promising targets are those with stable pulsations (in terms of period, amplitude and phase), short periods (to reduce the minimum detectable mass), several independent periods and relatively large amplitudes. To have several independent periods is important as it permits to check whether the same periodic pattern produced by a planet is present in all the periods (see Dalessio et al. 2010 for an example where this is not the case !), helping to exclude false detections due to other periodic effects (e.g. beating of close frequencies). SdB pulsators fit well these constraints and, due to the relatively short periods, are the best candidates. Other potential targets are the Delta Scuti stars, $\beta$ Cephei and RR Lyrae. In the next pages we show the results of a few preliminary tests on these pulsating stars using Kepler and CoRoT data, including also an eclipsing binary with a pulsating sdB component. The Kepler data were retrieved from the KASC data base (KASC is the Kepler AsteroSeismic Consortium, Gilliland et al. 2010). The CoRoT public data were downloaded from the CoRoT archive at LAEFF (Laboratory for Space Astrophysics and Theoretical Physics) in Spain.

\section{Subdwarf B stars}

Before the Kepler observations, the oscillating sdB stars could be divided into two groups: the short-period (p-mode) pulsators (or $\mathrm{sdBV}_{\mathrm{r}}=$ rapid pulsators) of the V361 Hya class from the prototype (Kilkenny et al. 1997), with effective temperatures between about 27,000 and 35,000 K, and the longer period (g-mode) pulsators (or $\mathrm{sdBV}_{\mathrm{s}}=$ slow pulsators) of the V1093 Her class (Green et al. 2003), with effective temperatures between $\sim 21,000$ and $\sim 28,000 \mathrm{~K}$. Four hybrid pulsators $\left(\mathrm{sdBV}_{\mathrm{rs}}\right)$ were known, showing both p- and g-modes at the same time (Schuh et al. 2006, see also Baran \& Fox-Machado 2010 for the complete list of hybrid pulsators before Kepler). This classification (compare also Kilkenny et al. 2010) may need some adjustment because the Kepler observations have shown that the temperature range of the two instability strips and the fraction of hybrid pulsators could be larger than previously believed.

In order to have a first feeling of what can be obtained with the timing technique on Kepler sdB data, we consider the star KIC 010139564, the only rapid sdB pulsator

\footnotetext{
${ }^{3}$ And in any case the timing method is not very sensitive to small orbital distances, see equation (1).
} 


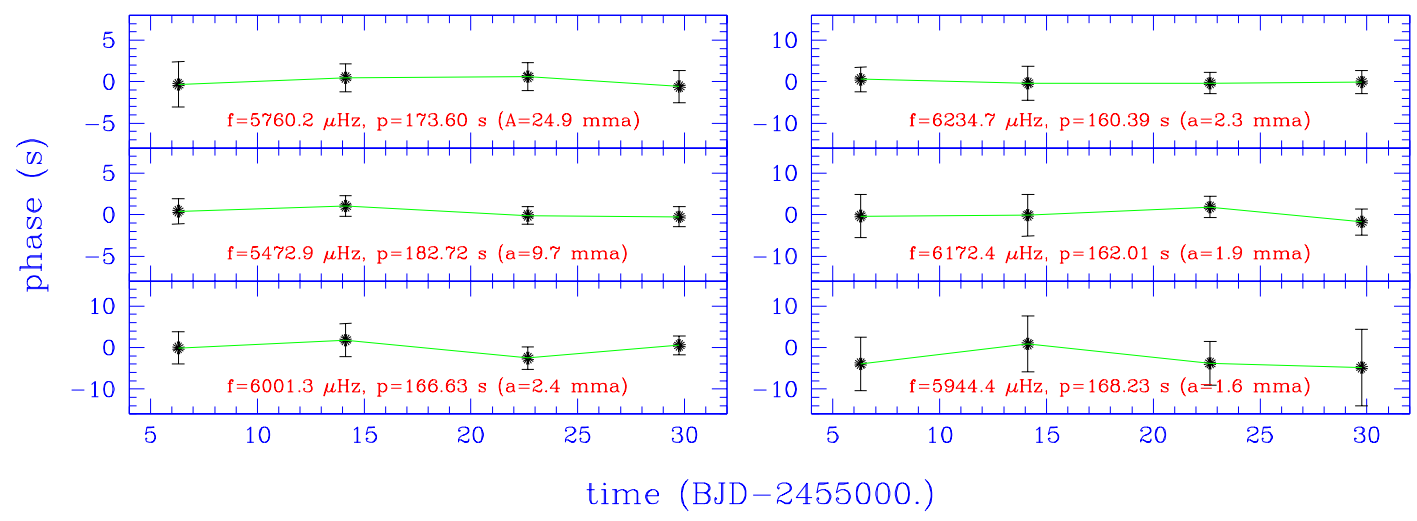

FIGURE 1. O-C plots of 6 pulsation periods of the short-period sdB pulsator KIC 10139564 (see Kawaler et al. 2010 for more details). Each point represents about 7.5 days of short cadence data. The phase stability of all these periods suggests that substellar companions can be detected. The small error bars of the two periods with higher amplitude (173.60 and $182.72 \mathrm{~s}$ ) allow to detect a planet of about $7 \mathrm{M}_{\mathrm{Jup}}$ at $1 \mathrm{AU}$ from the star. Using longer subsets, this limit can be reduced to about $2 \mathrm{M}_{\mathrm{Jup}}$ and even further (see text for more details).

observed by Kepler (Kawaler et al. 2010), and one of the best candidates to search for planets. Figure 1 shows the $\mathrm{O}-\mathrm{C}$ plot of six pulsation frequencies: these frequencies are considered also in Figs. 4 and 5 of Kawaler et al. (2010).

First of all, we see from Fig. 1 that the frequencies have in general stable phases. This is true not only for these six frequencies but also for almost all the other frequencies of this star, although they are not represented here. By comparing Fig. 1 with Fig. 4 of Kawaler et al. (2010), we see that, for most of these frequencies, the error bars are smaller by a factor of $(7.5 / 2)^{0.5} \simeq 1.9$, which is exactly what we expect considering that each point in Fig. 1 represents $\sim 7.5$ days of data while in Kawaler's plot each point corresponds to 2 days. However, at least one frequency at $5760.2 \mu \mathrm{Hz}$ behaves differently: the error bar of Fig. 1 is larger than the one obtained by Kawaler et al. (2010). The reason is in their Fig. 5, where we see that both phase and amplitude are changing periodically with a phase difference of about 90 degrees, suggesting that beating between closely spaced stable oscillation frequencies is occuring. From the smaller error bars of Fig. 1 we can estimate the minimum mass of a detectable planet: we obtain about 7 $\mathrm{M}_{\text {Jup }}$ for an orbital distance of $1 \mathrm{AU}$. However, potentially, the minimum mass can be reduced to about $2 \mathrm{M}_{\text {Jup }}$ using longer subsets of about 3 months each. This limit could be further extended to about $0.7 \mathrm{M}_{\text {Jup }}$ extrapolating from the small error bars in Fig. 4 of Kawaler et al. (2010), provided that the new Kepler data that will be collected in the next months/years will actually allow to separate the close frequencies and resolve the amplitude spectrum of this star.

For the long-period sdB pulsators, the first observations have shown that in general the phases are stable allowing to use the timing method also for these stars. For them, the minimum detectable mass of a substellar companion should be $\approx 10-50$ times higher than the numbers reported above considering that the periods are at least one order of 


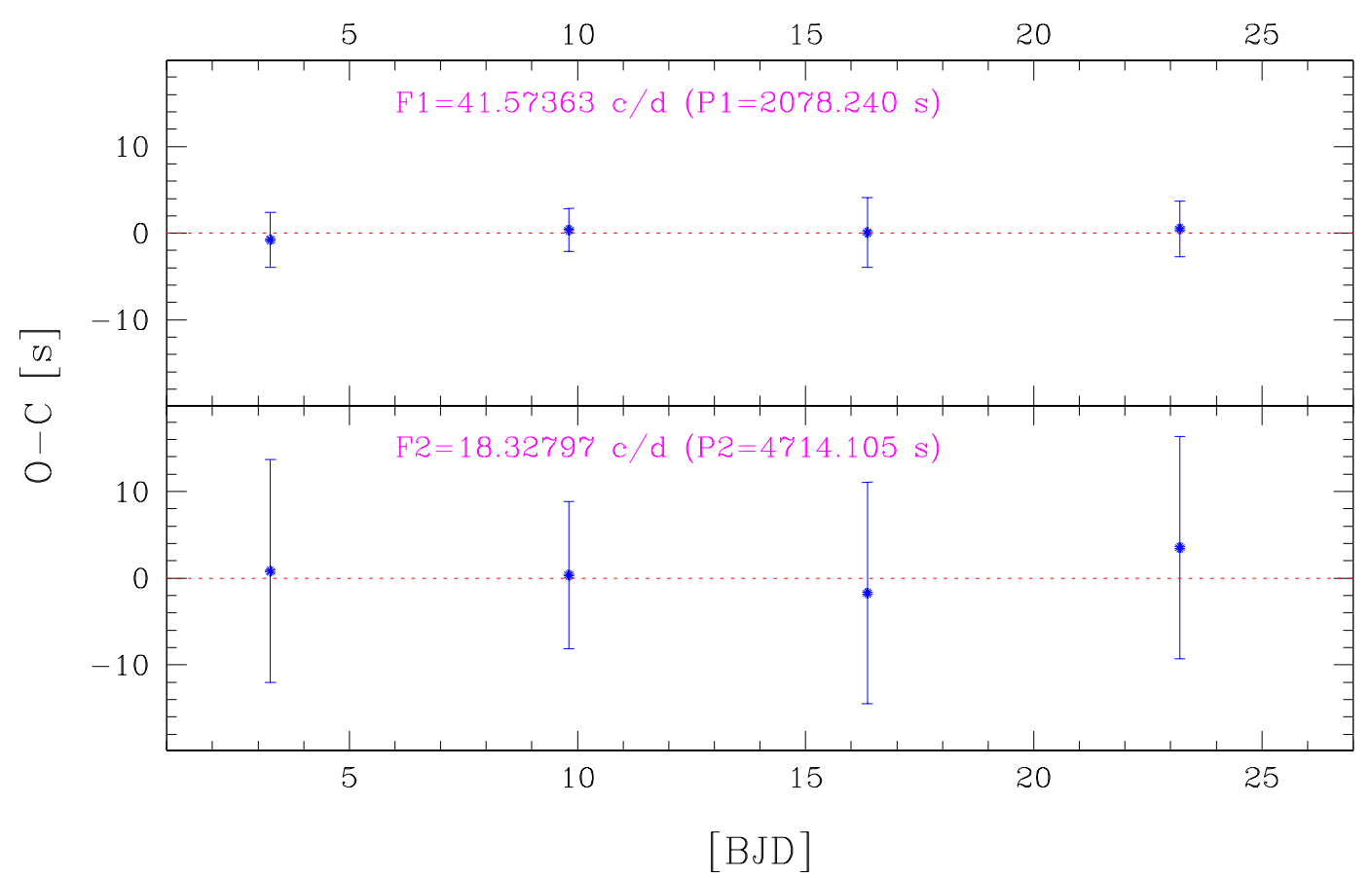

FIGURE 2. An example of O-C plots of a Delta Scuti star observed by Kepler. Each point represents about 7 days of short cadence data. The phase errors of the two main pulsation frequencies F1 and F2, of the order of \pm 4 and \pm 10 s respectively, suggest that Delta Scuti stars can be stable enough to use the timing technique. The fractional amplitudes of the two frequencies are $0.25 \%$ and $0.17 \%$ respectively.

magnitude longer. However, some of these stars show also a few peaks at high frequency, that are probably due to a few excited p-modes (Reed et al. 2010, Charpinet et al. 2011). If these short-period p-modes will be confirmed by the future Kepler observations, they could bring down again the minimum detectable masses of the potential companions.

\section{Delta Scuti stars}

Another interesting class of pulsators are the Delta Scuti stars. Although the periods are relatively long and comparable with those of the long-period sdB pulsators, their amplitudes can be larger, allowing to reduce the timing errors to a few seconds (see equation 2). An example of a Delta Scuti star with a relatively simple amplitude spectrum (not too many frequencies) and short periods is given in Fig. 2, where we see that the phases are stable, at least during the 1-month Kepler run in short cadence.

\section{3. $\beta$ Cephei stars}

The timing technique can also be used for $\beta$ Cephei stars. Here we show an example on the $\beta$ Cephei star HD 180642 (V1449 Aquilae), illustrating that these stars also may have stable phases. 


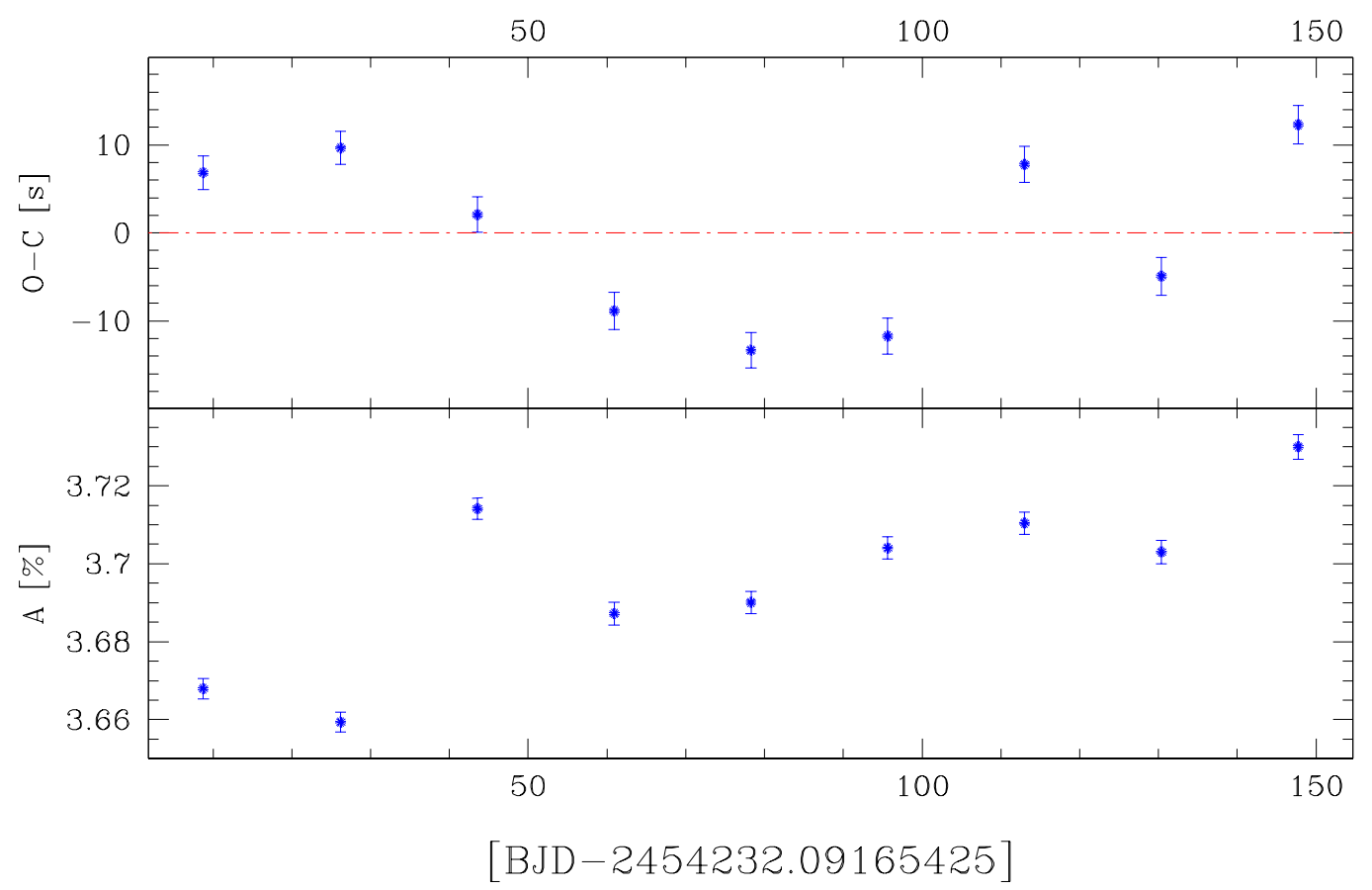

FIGURE 3. The upper and lower panels represent the phase and (fractional) amplitude variations, respectively, of the main pulsation frequency $\mathrm{F}=5.486889 \mathrm{c} / \mathrm{d}(\mathrm{P}=4.374063 \mathrm{~h})$ of the $\beta$ Cephei star HD 180642 (V1449 Aquilae). Each point represents about 17.4 days of the CoRoT long run LRc01 (156 days). The two panels suggest that phase and amplitude variations are not correlated and therefore these variations can not be attributed to beating of close frequencies. See Degroote et al. (2009) for more details on the pulsational behavior of this star.

HD 180642 was observed by the CoRoT satellite during 156 days of the long run LRc01, with a sampling cadence of $32 \mathrm{~s}$. The CoRoT data were analyzed in detail by Degroote et al. (2009), who found a complex amplitude spectrum with several combination frequencies. We applied a least-square sinusoidal fit to the CoRoT data using 15 frequencies obtained from pre-whitening, including a few combinations. In Figure 3 (upper panel) a preliminary O-C plot shows an apparent phase variation of the order of $20 \mathrm{~s}$ in 2 months, whose origin and nature is not clear. If this effect were due to a secular variation of the pulsation period, we would obtain $\dot{\mathrm{P}} \approx 4 \times 10^{-9} \mathrm{~s} / \mathrm{s}$. This number is significantly larger than the value found by Degroote et al. (2009) from direct measurement: about $1.3 \times 10^{-9} \mathrm{~s} / \mathrm{s}$. This difference deserves further study.

\section{RR Lyrae stars}

From the KASC data base we selected two RR Lyrae stars with the longest observational coverage (about 6 months). The O-C diagram of these RR Lyrae stars, shown in Fig. 4, indicates that the phase errors are generally large and, even more important, they are highly inhomogeneous. The reason of this behaviour is not clear. Although before the 


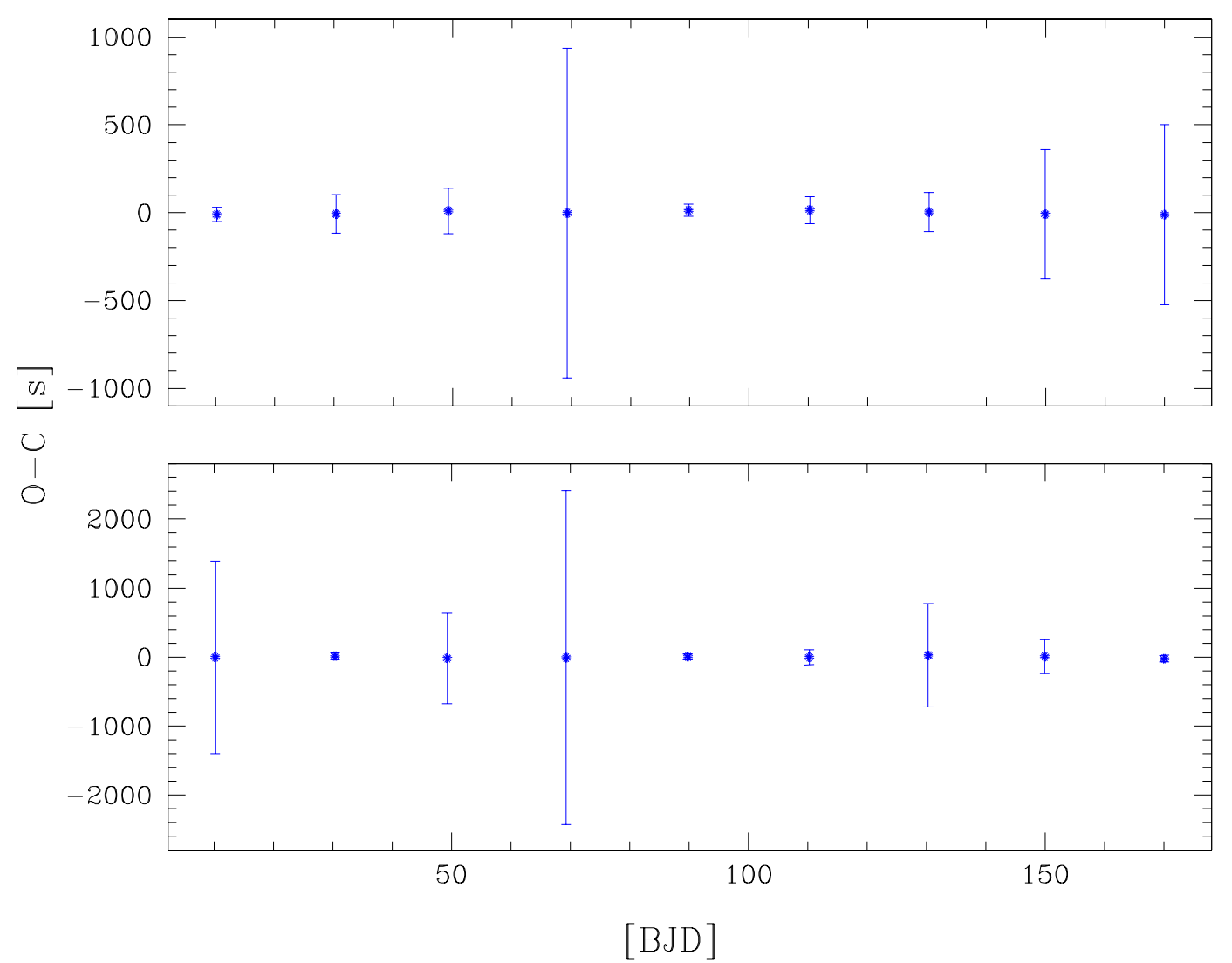

FIGURE 4. O-C diagram of two RR Lyrae stars observed by Kepler in long cadence (a measurement each $30 \mathrm{~min}$ ). Each point represents about 20 days of data. The vertical scale is different in each panel. The reason of the large and inhomogeneous phase errors is not clear (see text for more comments).

Kepler observation, both stars were considered as non-Blazhko stars, the non-uniform error distribution might be due to a low-level Blazhko modulation of phases and/or frequencies. Noise due to convection might also play a role. Although a deeper testing on a larger number of RR Lyrae stars is needed before drawing conclusions, this first example suggests that RR Lyrae might not be stable enough for the timing technique.

\section{Eclipse timing}

In this section we show an example of eclipse timing. We note that this method is related to the search for transit timing variations. The target, 2M1938+4603 (or KIC 09472174), is of particular interest because it is a $\mathrm{sdB}+\mathrm{dM}$ eclipsing binary system and the $\mathrm{sdB}$ component shows a very rich pulsational spectrum with periods ranging from $\sim 4 \mathrm{~min}$ to 5.5 hours. The orbital period of the system is $0.12576530 \mathrm{~d}$. For more details on 2M1938+4603 we refer the reader to the recent article by Østensen et al. (2010a). The 


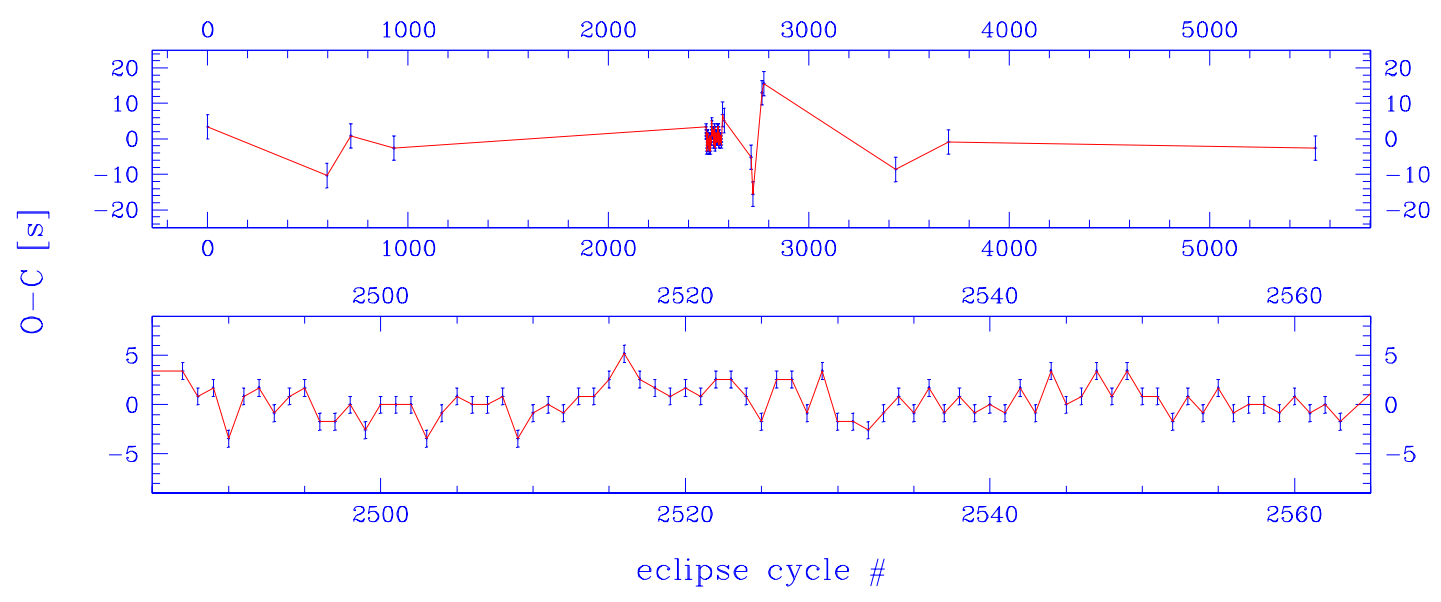

FIGURE 5. O-C diagram of the eclipsing sdB+dM binary 2M1938+4603 (KIC 09472174), based on eclipse timing. The times of the primary minima are taken from Østensen et al. (2010a). With a binary period of $0.12576530 \mathrm{~d}$, the upper panel corresponds to 695 days of ground based eclipse timings, with the Kepler 9.7-day commissioning data around cycle 2500. The latter are shown in more detail in the lower panel. The noise in the lower panel (and the detectable planetary mass) can be reduced by fitting several eclipses at once. To have an idea of the realistic timing errors, if we consider all the 77 Kepler eclipses in the lower panel, we obtain an $\mathrm{O}-\mathrm{C}$ standard deviation of $1.8 \mathrm{~s}$. With a 2 years (or longer) observation, and using 2.5 months of data for each point in the $\mathrm{O}-\mathrm{C}$ plot, the $4 \sigma$ detection limit would be reduced to about $4 \mathrm{M}_{\mathrm{Jup}}$ considering an orbital distance of $1 \mathrm{AU}$ from the binary. Potentially, the presence of a planet could be independently confirmed also from the pulsations of the sdB star.

eclipsing binary systems with a pulsating component, like this one, have the advantage of having two independent clocks given by the eclipse timing and by the pulsations (provided that there is no coupling between them). For this reason they are an ideal test case to verify the potential of the timing method in finding low-mass substellar objects.

\section{CONCLUSIONS AND FUTURE PERSPECTIVES}

In the last three years, the first discoveries of planets orbiting evolved stars after the red giant branch have proven that the timing method is an efficient way to detect substellar objects around compact stars, using either the stellar pulsation or the eclipse timing as a clock. This method, which is sensitive to Jupiter-mass planets in large orbits of $\approx 1$ $\mathrm{AU}$ or more, requires long-time photometric monitoring with frequent, or, better still, continuous, runs. In order to validate the method, it would be important to confirm these discoveries with other independent techniques. This in general is not easy because the targets are generally faint and many have spectral lines broadened by high gravity.

The high quality data from the CoRoT and Kepler satellites represent a unique opportunity due to the exceptional duty cycle. Kepler has the additional advantage of longer runs, up to 3.5 years. Preliminary tests show that sdB stars are the best candidates, in particular the short-period pulsators. Other good candidates are the Delta Scuti stars, 
at least those with a relatively simple amplitude spectrum (not too many frequencies). An example on a $\beta$ Cephei star shows that also these stars may have sufficiently stable phases, although the higher stellar mass implies a higher minimum mass of the potential substellar companions. RR Lyrae stars are more problematic and our preliminary tests suggest that phases and/or frequencies may have significant variations on time scales of weeks. An example of an eclipsing binary is given and shows that also for these targets Jovian planets can be detected. Moreover, if the binary system has a pulsating component like $2 \mathrm{M} 1938+4603$, the pulsations can be used, potentially, as an independent clock to confirm the detections.

\section{ACKNOWLEDGMENTS}

R.S. acknowledges financial support from the organizers, who waived the conference fees.

\section{REFERENCES}

M. Auvergne, P. Bodin, L. Boisnard et al., Astronomy \& Astrophysics, 506, 411-424 (2009).

A. Baran, L. Fox-Machado, Astroph. \& Space Science, 329, 193-197 (2010).

K. Beuermann, F. V. Hessman, S. Dreizler et al., Astronomy \& Astrophysics, 521, L60-L64 (2010a).

K. Beuermann, J. Buhlmann, J. Diese, et al., Astronomy \& Astrophysics, in press (arXiv:1011.3905) (2010b).

J. Bilíková, Y.-H. Chu, K. Y.-L. Su, R. A. Gruendl, Th. Rauch, "Spectral Analysis of Mid-IR Excesses of WDs", in Planetary Systems beyond the Main Sequence, edited by S. Schuh, H. Drechsel and U. Heber, AIP Conference Proceedings, American Institute of Physics, New York (these proceedings).

W. J. Borucki, D. Koch, G. Basri et al., Science 327, 977-980 (2010).

M. Breger, G. Handler, R. Garrido et al., Astronomy \& Astrophysics 349, 225-235 (1999).

S. Charpinet, V. Van Grootel, G. Fontaine, et al., Astronomy \& Astrophysics, submitted (2011).

Y. H. Chu, R. A. Gruendl, J. Bilíková, A. Riddle, K. Y.-L. Su, "Spitzer Surveys of IR Excesses of White Dwarfs", in Planetary Systems beyond the Main Sequence, edited by S. Schuh, H. Drechsel and U. Heber, AIP Conference Proceedings, American Institute of Physics, New York (these proceedings).

J. E. S. Costa, S. O. Kepler, Astronomy \& Astrophysics 489, 1225-1232 (2008).

J. Dalessio, J. L. Provencal, H. S. Shipman, "Searching for Planets with White Dwarf Pulsations: Spurious Detections" in Planetary Systems beyond the Main Sequence, edited by S. Schuh, H. Drechsel and U. Heber, AIP Conference Proceedings, American Institute of Physics, New York (these proceedings).

P. Degroote, M. Briquet, C. Catala et al., Astronomy \& Astrophysics, 506, 111-123 (2009).

S. Desidera, M. Barbieri, Astronomy \& Astrophysics 462, 345-353 (2007).

L. R. Doyle, H. J. Deeg,Proc. of the IAU Symp. 213, 80-84 (2002).

J. Farihi, "Evidence for Terrestrial Planetary System Remnants at White Dwarfs", in Planetary Systems beyond the Main Sequence, edited by S. Schuh, H. Drechsel and U. Heber, AIP Conference Proceedings, American Institute of Physics, New York (these proceedings).

B. T. Gänsicke, T. R. Marsh, J. Southworth, A. Rebassa-Mansergas, Science 314, 1908-1910 (2006).

B. T. Gänsicke, "Gaseous Debris Disks around WDs", in Planetary Systems beyond the Main Sequence, edited by S. Schuh, H. Drechsel and U. Heber, AIP Conference Proceedings, American Institute of Physics, New York (these proceedings).

S. Geier, H. Edelmann, U. Heber, L. Morales-Rueda, Astroph. Journal 702, L96-L99 (2009).

R. L. Gilliland, T. M. Brown, J. Christensen-Dalsgaard et al., PASP 122, 131-143 (2010).

E. M. Green, G. Fontaine, M. D. Reed et al., Astroph. Journal 583, L31-L34 (2003).

Z. Han, P. Podsiadlowski, P. F. L. Maxted, T. R. Marsh, N. Ivanova, MNRAS 336, 449-466 (2002).

Z. Han, P. Podsiadlowski, P. F. L. Maxted, T. R. Marsh, MNRAS 341, 669-691 (2003).

U. Heber, Annual Review of Astronomy \& Astrophysics 47, 211-251 (2009). 
F. V. Hessman, K. Beuermann, S. Dreizler, T. R. Marsh, S. G. Parsons, C. M. Copperwheat, D. E. Winget, G. F. Miller, J. J. Hermes, M. R. Schreiber, W. Kley, V. S. Dhillon, S. P. Littlefair, ’The Planets around the Post-Common Envelope Binary NN Serpentis" in Planetary Systems beyond the Main Sequence, edited by S. Schuh, H. Drechsel and U. Heber, AIP Conference Proceedings, American Institute of Physics, New York (these proceedings).

V. A. Jacobs, R. H. Østensen, H. Van Winckel, S. Bloemen, P. I. Pápics, G. Raskin, J. Debosscher, S. Uttenthaler, E. Van Aarle, C. Waelkens, E. Bauwens, T. Verhoelst, C. Gielen, H. Lehmann, R. Oreiro, "HERMES High-Resolution Spectroscopy of HD 149382: Where did the Planet go?", in Planetary Systems beyond the Main Sequence, edited by S. Schuh, H. Drechsel and U. Heber, AIP Conference Proceedings, American Institute of Physics, New York (these proceedings).

S. D. Kawaler, M. D. Reed, A. C. Quint et al., MNRAS, in press (arXiv: 1008.2356) (2010).

S. O. Kepler, J. E. S. Costa, B. G. Castanheira et al., Astroph. Journal 634, 1311-1318 (2005).

S. O. Kepler, D. E. Winget, R. E. Nather et al., Astroph. Journal 378, L45-L48 (1991).

D. Kilkenny, C. Koen, D. O’Donoghue, R. S. Stobie, MNRAS 285, 640-644 (1997).

D. Kilkenny, G. Fontaine, E. M. Green, S. Schuh, IBVS 5927, 1-3 (2010).

M. Konacki, A. Wolszczan, Astroph. Journal 591, L147-L150 (2003).

J. W. Lee, S.-L. Kim, C.-H. Kim et al., Astron. Journal 137, 3181-3190 (2009).

R. Lutz, S. Schuh, R. Silvotti, "The EXOTIME Targets HS 0702+6043 and HS 0444+0458”, in Planetary Systems beyond the Main Sequence, edited by S. Schuh, H. Drechsel and U. Heber, AIP Conference Proceedings, American Institute of Physics, New York (these proceedings).

P. F. L. Maxted, R. Napiwotzki, P. D. Dobbie, M. R. Burleigh, Nature 442, 543-545 (2007).

M. H. Montgomery, D. O’Donoghue, Delta Scuti Stars Newsletter 13, pp.28-32 (1999).

F. Mullally, D. E. Winget, S. Degennaro et al., Astroph. Journal 676, 573-583 (2008).

F. Mullally, W. T. Reach, S. Degennaro, A. Burrows, Astroph. Journal 694, 327-331 (2009).

R. H. Østensen, E. M. Green, S. Bloemen et al., MNRAS 408, L51-L55 (2010a).

R. H. Østensen, R. Silvotti, S. Charpinet et al., MNRAS, in press (arXiv:1007.3170) (2010b).

R. H. Østensen, R. Silvotti, S. Charpinet et al., MNRAS, submitted (2010c).

S. J. O'Toole, U. Heber, S. Geier, L. Classen, O. de Marco, "Radial Velocity Search for Substellar Companions to sdB Stars" in Planetary Systems beyond the Main Sequence, edited by S. Schuh, H. Drechsel and U. Heber, AIP Conference Proceedings, American Institute of Physics, New York (these proceedings).

M. Paparó, B. Szeidl, H. A. Mahdy, Astroph. \& Space Science 149, 73-82 (1988).

H. Perets, "Planets in Evolved Binary Systems", in Planetary Systems beyond the Main Sequence, edited by S. Schuh, H. Drechsel and U. Heber, AIP Conference Proceedings, American Institute of Physics, New York (these proceedings).

J. L. Provencal, "White Dwarfs and Planetary Systems", in Planets Beyond the Solar System and the Next Generation of Space Missions, edited by D. Soderblom, ASP Conference Series 119, pp. 123-126 (1997).

S.-B. Qian, L.-Y. Zhu, S. Zola et al., Astroph. Journal 695, L163-L165 (2009a).

S.-B. Qian, Z.-B. Dai, W.-P. Liao et al., Astroph. Journal 706, L96-L99 (2009b).

S.-B. Qian, W.-P. Liao, L.-Y. Zhu, Z.-B. Dai, Astroph. Journal 708, L66-L68 (2010a).

S.-B. Qian, W.-P. Liao, L.-Y. Zhu et al., MNRAS 401, L34-L38 (2010b).

W. T. Reach, M. J. Kuchner, T. von Hippel et al., Astroph. Journal 635, L161-L164 (2005).

M. D. Reed, S.D. Kawaler, R. H. Østensen et al., MNRAS, in press (arXiv:1008.0582).

S. Schuh, J. Huber, S. Dreizler et al. Astron. \& Astrophysics 445, L31-L34 (2006).

S. Schuh, R. Silvotti, R. Lutz et al. Astrophys. \& Space Science 329, 231-242 (2009).

S. Schuh, Astronomical Notes 331, 489-501 (2010).

R. Schwarz, A. D. Schwope, J. Vogel, et al., Astron. \& Astrophysics 496, 833-840 (2009).

J. Setiawan, R. Klement, Th. Henning, H.-W. Rix, B. Rochau, T. Schulze-Hartung, J. Rodmann, "A Planetary Companion around a Metal-Poor Star with Extragalactic Origin", in Planetary Systems beyond the Main Sequence, edited by S. Schuh, H. Drechsel and U. Heber, AIP Conference Proceedings, American Institute of Physics, New York (these proceedings).

J. Setiawan, R. Klement, Th. Henning, H.-W. Rix, B. Rochau, J. Rodmann, T. Schulze-Hartung, Science, published online (10.1126/science.1193342) (2010b).

S. Sigurdsson, H. B. Richer, B. M. Hansen, I. H. Stairs, S. E. Thorsett, Science 301, 193-196 (2003).

R. Silvotti, A. Bonanno, S. Bernabei et al., Astron. \& Astrophysics 459, 557-564 (2006). 
R. Silvotti, S. Schuh, R. Janulis et al., Nature 449, 189-191 (2007).

N. Soker, Astron. Journal 116, 1308-1313 (1998).

C. Sterken, ASP Conf. Series 335, 3-23 (2005).

K. Y. L. Su, Y.-H. Chu, G. H. Rieke et al., Astroph. Journal 657, L41-L45 (2007).

S. E. Thorsett, Z. Arzoumanian, F. Camilo, A. G. Lyne, Astroph. Journal 523, 763-770 (1999).

E. Villaver, M. Livio, Astroph. Journal 705, L81-L85 (2009).

D. T. Wickramasinghe, J. Farihi, C. A. Tout, L. Ferrario, R. J. Stancliffe, MNRAS 404, 1984-1991 (2010).

A. Wolszczan, D. A. Frail, Nature 355, 145-147 (1992). 